\title{
Scale-similar models for large-eddy simulations
}

\author{
F. Sarghini, U. Piomelli, ${ }^{\text {a) }}$ and E. Balaras \\ Department of Mechanical Engineering, University of Maryland, College Park, Maryland 20742
}

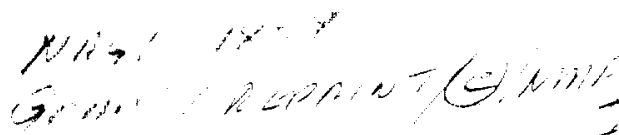

(Received 23 October 1998; accepted 1 March 1999)

Scale-similar models employ multiple filtering operations to identify the smallest resolved scales, which have been shown to be the most active in the interaction with the unresolved subgrid scales. They do not assume that the principal axes of the strain-rate tensor are aligned with those of the subgrid-scale stress (SGS) tensor, and allow the explicit calculation of the SGS energy. They can provide backscatter in a numerically stable and physically realistic manner, and predict SGS stresses in regions that are well correlated with the locations where large Reynolds stress occurs. In this paper, eddy viscosity and mixed models, which include an eddy-viscosity part as well as a scale-similar contribution, are applied to the simulation of two flows, a high Reynolds number plane channel flow, and a three-dimensional, nonequilibrium flow. The results show that simulations without models or with the Smagorinsky model are unable to predict nonequilibrium effects. Dynamic models provide an improvement of the results: the adjustment of the coefficient results in more accurate prediction of the perturbation from equilibrium. The Lagrangian-ensemble approach [Meneveau et al., J. Fluid Mech. 319, 353 (1996)] is found to be very beneficial. Models that included a scale-similar term and a dissipative one, as well as the Lagrangian ensemble averaging, gave results in the best agreement with the direct simulation and experimental data. (c) 1999 American Institute of Physics.

[S1070-6631(99)03306-1]

\section{INTRODUCTION}

In the large-eddy simulation (LES) approach all flow variables are decomposed into a resolved, large-scale, component, and an unresolved part due to small scales, which is modeled. Governing equations for the large-scale variables can be derived by the application of a spatial filter (denoted by an overbar) of characteristic width $\bar{\Delta}$ to the continuity and Navier-Stokes equations. The effect of the small scales upon the resolved part of turbulence appears in the subgrid-scale (SGS) stresses. The success of the LES approach depends to a large extent on the accurate representation of this term.

Since the small scales tend to be more homogeneous and isotropic than the large ones, they can be modeled using simpler parameterizations than their counterparts in the Reynolds-averaged Navier-Stokes (RANS) equations; furthermore, since the resolved scales are responsible for a significant proportion of the Reynolds stresses, modeling errors should not be as significant. For these reasons, and also to avoid increasing the cost of the calculations, eddy-viscosity type models ${ }^{1}$ are used for the parameterization of the SGS stresses.

Although these models were found to give a poor prediction of the SGS stresses on a local level, ${ }^{2,3}$ they predict the global dissipation fairly accurately; this may be one reason for their success and widespread use. Many of the deficiencies of eddy-viscosity models (incorrect limiting behavior near solid boundaries, nonvanishing eddy-viscosity in

\footnotetext{
${ }^{a)}$ Author to whom correspondence should be addressed. Telephone: (301) 405-5254; Fax: (301) 314-9477; Electronic mail: ugo@eng.umd.edu
}

laminar regions) can be avoided either by introducing ad hoc modifications (van Driest damping, ${ }^{4}$ intermittency functions, ${ }^{5}$ or low-Reynolds number corrections, ${ }^{6}$ for instance) or adjusting dynamically the model coefficients. ${ }^{7,8}$ Two fundamental drawbacks of these models, however, cannot be easily corrected: Their purely dissipative character, and the assumption that the principal axes of the strain-rate and SGS stress tensors are aligned.

While, on average, the small scales drain energy from the large ones, reversed energy transfer (backscatter) is known to occur locally, and be significant, even in equilibrium flows. Theoretical ${ }^{9}$ arguments, as well as numerical ${ }^{10}$ and experimental ${ }^{11}$ studies, have shown that the net energy transfer is given by the difference of two terms, forward and backward scatter, and that each is much larger than their difference. Algebraic eddy-viscosity models cannot account for backscatter, since the negative eddy viscosity required to yield a negative energy transfer results in numerical instability in the form of exponentially growing components of the solution. Physically, one would expect backscatter to deplete the energy available to the subgrid scales, and reduce the magnitude of the eddy viscosity to zero on a time scale characteristic of the small scales, rapidly re-establishing the equilibrium. Algebraic eddy-viscosity models, however, cannot follow this physical scenario since their time scale, usually the reciprocal of the large-scale strain-rate, depends on the largest scales of the flow. The addition of an equation for the SGS energy can overcome this difficulty, ${ }^{12}$ at the expense of increased cost of the calculation.

Furthermore, in a priori investigations of interscale energy transfer in homogeneous and wall-bounded flows, 
Domaradzki and co-workers ${ }^{13,14}$ have shown that the interscale energy transfer is mainly due to interactions involving modes within one octave of the cutoff wavenumber. Nonlocal energy transfer (which can be represented by eddyviscosity models) was found to be less significant. This indicated that there might be some correlation of the SGS stresses with the Reynolds stresses (or, at least, with the part of the Reynolds stresses due to the smallest resolved scales). This conjecture was confirmed by Piomelli et al., ${ }^{15}$ who observed significant correlation between large-scale, energy(and Reynolds-stress-) producing events, and energy transfer to and from the small scales.

These results supply a posteriori justification for the use of scale-similar and mixed models, that predict the SGS stresses based on the smallest resolved scales. The first model of this type was proposed by Bardina et al., ${ }^{16}$ who performed a priori tests and calculations of homogeneous isotropic turbulence using a mixed model, which included a scale-similar as well as an eddy-viscosity term to account for the dissipation by the small scales. They obtained improved results, compared with the standard Smagorinsky model. ${ }^{1} A$ priori tests ${ }^{11,16}$ have shown that scale-similar and mixed models give higher correlation coefficients between the exact and modeled stresses than the Smagorinsky model. Furthermore backscatter may occur in a numerically stable manner.

Piomelli et al. ${ }^{3}$ found that the choice of model must be coupled with that of the filter, and that an inconsistent choice gives inaccurate results. When the Gaussian filter is used and the Leonard stresses are computed, for instance, inclusion of the scale-similar part of the model gives more accurate results than the eddy-viscosity part alone. Horiuti ${ }^{17}$ observed that the major role of the scale-similar part of the model is to provide the backscatter of SGS energy.

Scale-similar models have been recently revisited in the framework of dynamic modeling ideas. A dynamic model including a scale-similar part has been applied by Zang et al. ${ }^{18}$ to the calculation of flow in a driven cavity. They found that the inclusion of the scale-similar part reduced the contribution of the Smagorinsky part substantially. This allowed them to use a local formulation of the dynamic model in which the coefficient was allowed to vary in space, rather than being averaged over homogeneous directions. ${ }^{7}$ The low Reynolds number of the calculation, however, made the effect of the SGS stresses less significant, and makes it difficult to generalize their findings. Vreman et al. ${ }^{19}$ reformulated this model in a consistent fashion, obtaining improved results in the LES of a mixing layer. Wu and Squires ${ }^{20}$ performed the large eddy simulation of an equilibrium three-dimensional turbulent boundary layer using a mixed Lagrangian model similar to that proposed by Zang et al. ${ }^{18}$ in the formulation by Vreman et al. ${ }^{19}$ The model coefficient was evaluated using the Lagrangian ensemble-averaging procedure. ${ }^{21}$

Liu et al. ${ }^{11}$ performed a detailed analysis of the SGS energy fluxes using experimental data at the far field of a round jet at $\operatorname{Re}_{\lambda}=310$. They decomposed the velocity field into logarithmic bands and found that the flow fields in consecutive bands are similar, and that $\tau_{i j}$ and the resolved turbulent stresses (which represent the stresses due to the small- est resolved scales), as well as their contractions with $\bar{S}_{i j}$ are well correlated. They then proposed a new scale-similar model in which the SGS stresses are assumed to be proportional to the resolved turbulent stresses $\mathcal{L}_{i j}$. This model, with the addition of a dissipative term, was applied by Anderson and Meneveau, ${ }^{22}$ within the framework of the Lagrangian-ensemble averaging, ${ }^{21}$ to the study of isotropic turbulence decay. It was found that the contribution of the similarity term to the turbulent kinetic energy is negligible, while its contribution to the SGS force (the divergence of the SGS stresses) is more then double that of the dissipative term.

Several additional dynamic versions of mixed models have been proposed and tested. ${ }^{23-25}$ Their application has, however, been almost entirely limited to equilibrium or nearequilibrium flows.

Purpose of this paper is to examine the behavior of scale-similar models both in equilibrium and nonequilibrium flows, and compare their performance with that of standard eddy-viscosity models. Two cases have been considered: The fully-developed flow in a plane channel, and the flow in a three-dimensional shear-driven boundary layer obtained by moving the lower wall of a fully-developed plane channel in the spanwise direction. The latter is of particular interest for SGS modeling, since the SGS stresses do not adjust instantaneously to the secondary shear, ${ }^{26}$ and the temporal response of the modeled SGS stresses can be expected to affect the results.

In the following, mixed models and ways to evaluate the model coefficients will be presented. Then, numerical results will be discussed. Finally, some conclusions will be drawn.

\section{PROBLEM FORMULATION}

\section{A. Governing equations and boundary conditions}

In LES the filtered and SGS quantities are defined as

$$
\bar{f}=\int_{D} f\left(\mathbf{x}^{\prime}\right) G\left(\mathbf{x}-\mathbf{x}^{\prime} ; \bar{\Delta}\right) d \mathbf{x}^{\prime}, \quad f^{\prime}=f-\bar{f},
$$

where $G$ is the filter function of characteristic width $\bar{\Delta}$, and the integral is extended to the entire domain $D$. The application of the filtering operation to the continuity and the Navier-Stokes equations gives the resolved Navier-Stokes equations, which, in incompressible flow, are

$$
\begin{aligned}
& \frac{\partial \bar{u}_{i}}{\partial t}+\frac{\partial}{\partial x_{j}}\left(\bar{u}_{i} \bar{u}_{j}\right)=-\frac{1}{\rho} \frac{\partial \bar{p}}{\partial x_{i}}-\frac{\partial \tau_{i j}}{\partial x_{j}}+\nu \nabla^{2} \bar{u}_{i}, \\
& \frac{\partial \bar{u}_{i}}{\partial x_{i}}=0,
\end{aligned}
$$

(where $p$ is the pressure, $\nu$ the kinematic viscosity, and $u_{1}$, $u_{2}$, and $u_{3}$, or $u, v$, and $w$, are the streamwise, wall-normal and spanwise components of velocity, respectively), which govern the dynamics of the large, energy-carrying scales of motion. The effect of the small scales upon the resolved part of turbulence appears in the subgrid-scale (SGS) stress term, $\tau_{i j}=\overline{u_{i} u_{j}}-\bar{u}_{i} \bar{u}_{j}$, which must be modeled. 


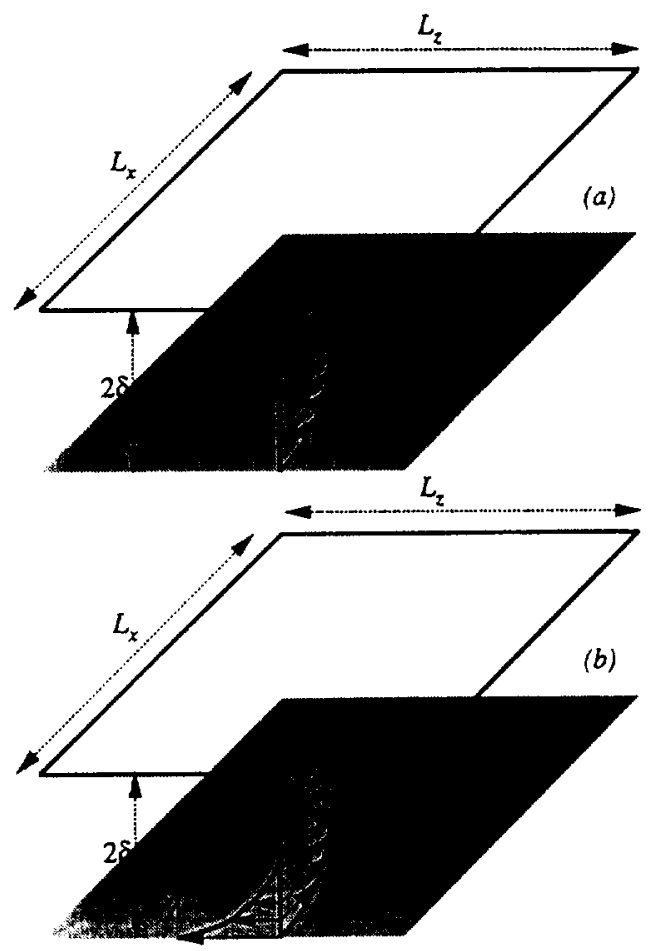

FIG. 1. Sketch of the physical configurations: (a) Plane channel; (b) threedimensional boundary layer.

Two cases were examined: A two-dimensional plane channel flow, at $\operatorname{Re}_{r}=\delta u_{\tau} / \nu=180$ and 1050 , and a threedimensional (3D) boundary layer. In all cases, periodic boundary conditions were applied in the streamwise and spanwise directions, while the no-slip conditions were used at the solid walls. The three-dimensional boundary layer is a temporally developing flow obtained by imposing an impulsive spanwise motion, with magnitude equal to $47 \%$ of the initial mean centerline velocity, to the wall of a fully developed plane channel flow; the initial condition was a calculation of fully developed plane channel flow at Reynolds number $\operatorname{Re}_{\tau}=180$. These parameters matched the DNS calculations of Coleman et al. ${ }^{27}$ The flow was allowed to develop until a collateral state (one in which the direction of the mean velocity is the same at each $y$ ) was reached. While in the direct numerical simulation (DNS) of Coleman et al. ${ }^{27}$ only one wall was set in motion, in the present case both walls were moved in opposite directions to double the statistical sample. It was verified that the important statistics were not affected up to a dimensionless time $t u_{\tau, o} / \delta=1.2$ (where $u_{\tau, o}$ is the value of the friction velocity before the walls are set into motion). The two configurations examined are sketched in Fig. 1.

\section{B. Numerical method}

The governing equations [Eqs. (2.2) and (2.3)] were integrated in time using a Fourier-Chebychev pseudo-spectral collocation scheme. ${ }^{28}$ The skew-symmetric form of the momentum equation [Eq. (2.2)] was employed, and the timeadvancement was performed by a fractional time step method with a semi-implicit scheme; the wall-normal diffusion term was advanced using the Crank-Nicolson scheme, and the remaining terms by a low-storage third-order Runge-Kutta scheme. No de-aliasing was performed.

The computational domain in the streamwise, wallnormal and spanwise directions was $4 \pi \delta \times 2 \delta \times 4 \pi \delta / 3$ (where $\delta$ is the channel half-width) for the low-Reynolds number case, $2 \pi \delta \times 2 \delta \times 2 \pi \delta$ for the high Reynolds number calculations, and $3 \pi \delta \times 2 \delta \times 8 \pi \delta / 3$ for the threedimensional case. The calculation parameters are summarized in Table I. For the sake of comparison, all quantities expressed in wall units in the Table are calculated using the experimental value of the friction velocity obtained from the correlation in the reference by Dean. ${ }^{29} \mathrm{~A}$ wider box and a finer mesh were used in the 3D boundary-layer calculation to account for the turning of the flow in the near wall, that causes the streaks to be reoriented at an angle to the $x$ axis. To resolve these structures, which are no longer aligned in the streamwise direction, a higher streamwise resolution is required.

\section{SUBGRID STRESS MODELS}

\section{A. Eddy viscosity, scale-similar, and mixed models}

Eddy-viscosity models parameterize the SGS stresses as

$$
\tau_{i j}-\frac{\delta_{i j}}{3} \tau_{k k}=-2 \nu_{T} \bar{S}_{i j}=-2 C_{e v} \Delta^{2}|\bar{S}| \bar{S}_{i j},
$$

in which $\delta_{i j}$ is Kronecker's delta and $|\bar{S}|=\left(2 \bar{S}_{i j} \bar{S}_{i j}\right)^{1 / 2}$ is the magnitude of the large-scale strain-rate tensor

$$
\bar{S}_{i j}=\frac{1}{2}\left(\frac{\partial \bar{u}_{i}}{\partial x_{j}}+\frac{\partial \bar{u}_{j}}{\partial x_{i}}\right) .
$$

Lilly ${ }^{30}$ determined the value of the coefficient $C_{e v}$ by assuming that the small scales are in equilibrium, and obtained $C_{e v} \approx 0.032 . C_{e v}$ can also be evaluated dynamically ${ }^{7.8}$ (see below).

Scale-similar models were introduced by Bardina et al. ${ }^{16}$ who assumed that the component of the SGS most active in the energy transfer from large to small scales can be estimated with sufficient accuracy from the smallest resolved

TABLE 1. Simulation parameters.

\begin{tabular}{llll}
\hline \hline & \multicolumn{1}{c}{$\mathrm{Re}_{r}=180$} & \multicolumn{1}{c}{$\mathrm{Re}_{r}=1050$} & 3D boundary layer \\
\hline Domain size (outer units) & $4 \pi \delta \times 2 \delta \times 4 \pi \delta / 3$ & $2 \pi \delta \times 2 \delta \times 2 \pi \delta$ & $3 \pi \delta \times 2 \delta \times 8 \pi \delta / 3$ \\
Domain size (wall units) & $2260 \times 360 \times 750$ & $6600 \times 2100 \times 3300$ & $1700 \times 360 \times 1500$ \\
Mesh points & $48 \times 65 \times 64$ & $64 \times 97 \times 128$ & $64 \times 65 \times 64$ \\
Grid size, $\Delta x^{+} \times \Delta y_{\min }^{+} \times \Delta z^{+}$ & $47 \times 0.2 \times 12$ & $103 \times 0.5 \times 26$ & $27 \times 0.2 \times 23$ \\
\hline
\end{tabular}


scales, which can be obtained by filtering the SGS velocity given in Eq. (2.1) as $\overline{u_{i}^{\prime}}=\bar{u}_{i}-\overline{\bar{u}}_{i}$. They then made the following assumptions:

$$
\begin{aligned}
& \overline{u_{i}^{\prime} u_{j}^{\prime}} \sim \overline{u_{i}^{\prime} u_{j}^{\prime}} \simeq\left(\bar{u}_{i}-\overline{\bar{u}}_{i}\right)\left(\bar{u}_{j}-\overline{\bar{u}}_{j}\right), \\
& \overline{u_{i}^{\prime} \bar{u}_{j}} \sim \overline{u_{i}^{\prime} \bar{u}_{j}} \simeq\left(\bar{u}_{i}-\overline{\vec{u}}_{i}\right) \overline{\bar{u}}_{j},
\end{aligned}
$$

to yield a model of the form

$$
\tau_{i j}=C_{s s}\left(\overline{\bar{u}_{i} \bar{u}_{j}}-\overline{\bar{u}}_{i} \overline{\bar{u}}_{j}\right) \text {, }
$$

where $C_{s s}$ is a model coefficient that must be set to one to recover Galilean invariance. ${ }^{31}$

The scale-similar model (3.5) was found to be insufficiently dissipative; therefore, it was combined with an eddyviscosity dissipative component to yield

$$
\tau_{i j}=\overline{\vec{u}_{i} \bar{u}_{j}}-\bar{u}_{i} \bar{u}_{j}-2 C_{e v} \bar{\Delta}^{2}\left|\bar{S}_{\mid}\right| \bar{S}_{i j} .
$$

Zang et al ${ }^{18}$ used a dynamic form of this model, in which the model coefficient, $C_{e v}$, was adjusted dynamically.

As mentioned above, Liu et al. ${ }^{11}$ proposed a model in which the SGS stresses are assumed to be proportional to the resolved turbulent stresses $\mathcal{L}_{i j}=\widehat{\bar{u}}_{i} \overline{\bar{u}}_{j}-\hat{\bar{u}}_{i} \hat{\bar{u}}_{j}$ (where $\hat{\imath}$ denotes the application of a filter with characteristic scale $\Delta>\bar{\Delta}$ ):

$$
\tau_{i j}=C_{s s}\left(\widehat{\bar{u}}_{i} \overline{\bar{u}}_{j}-\hat{\bar{u}}_{i} \hat{\bar{u}}_{j}\right) \text {, }
$$

where $C_{s s}$ is a coefficient of order one. ${ }^{11}$ An eddy viscosity term can be added the scale-similar model (3.7) to yield

$$
\tau_{i j}=C_{s s}\left(\widehat{\bar{u}}_{i} \overline{\bar{u}}_{j}-\hat{\bar{u}}_{i} \hat{\bar{u}}_{j}\right)-2 C_{e v} \bar{\Delta}^{2}|\bar{S}| \bar{S}_{i j} .
$$

\section{B. Dynamic coefficient adjustment}

Dynamic adjustments of the model coefficient has been shown to be extremely beneficial: The models become more sensitive to the local state of the flow, resulting in more accurate prediction of transition or re-laminarization, better near-wall behavior, and altogether more accurate results than models in which the coefficients are specified a priori. For this reason several of the models described in the previous section have been implemented dynamically.

Dynamic adjustment of the model coefficients is based on the identity ${ }^{32}$

$$
\mathcal{L}_{i j}=\widehat{\bar{u}_{i}} \bar{u}_{j}-\hat{\bar{u}}_{i} \hat{\bar{u}}_{j}=T_{i j}-\widehat{\tau_{i j}},
$$

which relates the resolved turbulent stresses $\mathcal{L}_{i j}$, the subgridscale stresses $\tau_{i j}$ and the subtest stresses $T_{i j}=\widehat{\overline{u_{i} u_{j}}}-\hat{\bar{u}}_{i} \hat{\bar{u}}_{j}$, obtained by applying a filter $\hat{\hat{G}}$, of characteristic length $\hat{\Delta}$, to the Navier-Stokes equations.

Consider an eddy-viscosity model, valid for both subgrid and subtest stresses, of the form

$$
\tau_{i j}=-2 C_{e v} \alpha_{i j}, \quad T_{i j}=-2 C_{e v} \beta_{i j},
$$

where $\alpha_{i j}$ and $\beta_{i j}$ are generic terms of the form shown in Eq. (3.1). Upon substituting Eq. (3.10) into Eq. (3.9), the identity can be satisfied only approximately, since the stresses are replaced by modeling assumptions, and the system is overdetermined (five independent equations are available to de- termine a single coefficient). Lilly ${ }^{8}$ proposed that the error incurred when a single coefficient is used be minimized in a least-square sense. The error is

$$
\begin{aligned}
e_{i j} & =\mathcal{L}_{i j}-T_{i j}+\widehat{\tau_{i j}} \\
& =\mathcal{L}_{i j}+2 C_{e v}\left(\beta_{i j}-\widehat{\alpha_{i j}}\right)=\mathcal{L}_{i j}+2 C_{e v} M_{i j},
\end{aligned}
$$

with $M_{i j}=\beta_{i j}-\widehat{\alpha_{i j}}$. The least-squares minimization procedure requires that $\partial E^{2} / \partial C_{e v}=\partial\left(e_{i j} e_{i j}\right) / \partial C_{e v}=0$, where the brackets indicate an appropriate average, ${ }^{33}$ this yields

$$
C_{e U}=-\frac{1}{2} \frac{P_{\mathcal{L} M}}{P_{M M}},
$$

where $P_{E F}=\left\langle E_{i j} F_{i j}\right\rangle$.

Consider now a one-coefficient mixed model of the form

$$
\tau_{i j}=A_{i j}-2 C_{e v} \alpha_{i j}, \quad T_{i j}=B_{i j}-2 C_{e v} \beta_{i j},
$$

where, once again, $A_{i j}$ and $B_{i j}$ represent generic forms of the scale-similar part of the stresses, as in Eq. (3.5) or Eq. (3.7). In this case the least-squares minimization procedure gives

$$
C_{e V}=-\frac{1}{2} \frac{P_{\mathcal{L} M}-P_{N M}}{P_{M M}}
$$

where $N_{i j}=B_{i j}-\widehat{A_{i j}}$.

An analogous method can be used in the case of two model coefficients: Let

$$
\tau_{i j}=C_{s s} A_{i j}-2 C_{e v} \alpha_{i j}, \quad T_{i j}=C_{s s} B_{i j}-2 C_{e v} \beta_{i j} .
$$

The error is

$$
e_{i j}=\mathcal{L}_{i j}-C_{s s} N_{i j}+2 C_{e v} M_{i j} .
$$

Requiring that $\partial E^{2} / \partial C_{s s}=\partial E^{2} / \partial C_{e v}=0$ results in

$$
\begin{aligned}
& C_{s s}(\mathrm{x}, t)=-\frac{P_{M N} P_{\mathcal{L}}-P_{M M} P_{\mathcal{L}}}{P_{M N} P_{M N}-P_{M M} P_{N N}}, \\
& C_{e U}(\mathrm{x}, t)=-\frac{1}{2} \frac{P_{M N} P_{\mathcal{~} N}-P_{N N} P_{L M}}{P_{M N} P_{M N}-P_{M M} P_{N N}} .
\end{aligned}
$$

\section{Ensemble averaging}

It remains to determine the ensemble-averaging operator $\langle\cdot\rangle$. The ensemble average has the purpose of removing very sharp fluctuations of the coefficient, which tend to destabilize numerical calculations and make the model inconsistent, since the model coefficients cannot be extracted from the filtering operation (i.e., the difference $\widehat{C \alpha_{i j}}-C \widehat{\alpha_{i j}}$ becomes significant). Germano et al. ${ }^{7}$ averaged the model coefficient over all homogeneous directions, thereby removing completely the mathematical inconsistency. Ghosal et al. ${ }^{12}$ performed no averaging; they, however, used an integral formulation of the identity (3.9) that rigorously removed the mathematical inconsistency at the expense of solving an integral equation at each time step (an expense comparable to the solution of a Poisson equation, therefore, significant). Localized filtering can be performed over the scale $\hat{\Delta}$ (somewhat justifiable by the consideration that, if the same coefficient is used to model both $\tau_{i j}$ and $T_{i j}$ it must be smooth on the test-filter scale). Zang et al. ${ }^{18}$ performed this type of av- 
eraging; the inclusion of the scale-similar part into their model decreased the contribution of the eddy-viscosity term, and no spuriously high values of the coefficient were observed.

An attractive alternative localized model uses the Lagrangian ensemble proposed by Meneveau et al. ${ }^{21}$ and defined as

$$
\mathcal{I}_{f}=\langle f\rangle=\int_{-\infty}^{t} f\left(t^{\prime}\right) W\left(t-t^{\prime}\right) d t^{\prime},
$$

where the integral is carried out following a fluid path-line, and $W(t)$ is an exponential weighting functions appropriately chosen to give more weight to recent times. The eddyviscosity model coefficient in Eq. (3.12), for instance, can then be written as

$$
C_{e v}=-\frac{1}{2} \frac{\left\langle\mathcal{L}_{i j} M_{i j}\right\rangle}{\left\langle M_{m n} M_{m n}\right\rangle}=-\frac{1}{2} \frac{\mathcal{I}_{L M}^{n}}{\mathcal{I}_{M M}^{n}},
$$

where a superscript $n$ denotes the time step, and

$$
\begin{aligned}
& \mathcal{I}_{L M}=\int_{-\infty}^{t} \mathcal{L}_{i j}\left(t^{\prime}\right) M_{i j}\left(t^{\prime}\right) W\left(t-t^{\prime}\right) d t^{\prime}, \\
& \mathcal{I}_{M M}=\int_{-\infty}^{t} M_{i j}\left(t^{\prime}\right) M_{i j}\left(t^{\prime}\right) W\left(t-t^{\prime}\right) d t^{\prime} .
\end{aligned}
$$

If the weight function is chosen to be

$$
W(t)=T^{-1} \exp (-t / T),
$$

where the time constant is given by $T=1.5 \Delta$ $\times\left(-8 \mathcal{I}_{L M}^{n} \mathcal{I}_{M M}^{n}\right)^{-1 / 8}$, these integrals can be approximated by

$\mathcal{I}_{L M}^{n}(\mathbf{x})=H\left\{\varepsilon \mathcal{L}_{i j}^{n} M_{i j}^{n}+(1-\varepsilon) \mathcal{I}_{L M}^{n-1}\left(\mathbf{x}-\mathbf{u}^{n} \Delta t\right)\right\}$,

$\mathcal{I}_{M M}^{n}(\mathbf{x})=H\left\{\varepsilon M_{i j}^{n} M_{i j}^{n}+(1-\varepsilon) \mathcal{I}_{M M}^{n-1}\left(\mathbf{x}-\mathbf{u}^{n} \Delta t\right)\right\}$,

where $H$ is the ramp function, the evaluation of the integrals at $\mathbf{x}-\mathbf{u}^{n} \Delta t$ can be performed by linear interpolation, and

$$
\varepsilon=\frac{\Delta t / T}{1+\Delta t / T}
$$

In order to avoid complex values for $T$, if $C_{e v}(\mathbf{x}, t)=0$ is reached, $I_{L M}^{n}$ is set to zero.

This type of averaging is based on the consideration that the memory effects should be calculated in a Lagrangian framework, following the fluid particle, rather than at an Eulerian point, which sees different particles, with different histories, at each instant. A more complete analysis of this model can be found in the reference by Meneveau et al. ${ }^{21}$

\section{RESULTS AND DISCUSSION}

\section{A. Models tested}

It was not the purpose of this paper to perform an exhaustive test of all scale-similar and mixed models presented in literature, but only to examine some representative ones, investigate their behavior, and especially their response to perturbation from equilibrium.
To compare mixed and eddy viscosity models, and evaluate the effect of the scale-similar part, the following models were tested:

(1) Smagorinsky model (SMG): Only the anisotropic part of the SGS stresses is modeled, the coefficient $C_{e v}$ is set to 0.01 , and van Driest ${ }^{4}$ damping is used to account for nearwall effects

$$
\tau_{i j}-\frac{\delta_{i j}}{3} \tau_{k k}=-2 C_{e v}\left[\Delta\left(1-e^{-y^{+} / A^{+}}\right)\right]^{2}|\bar{S}| \bar{S}_{i j},
$$

where $y^{+}=u_{\tau}(1-|y| / \delta) / \nu$, and $A^{+}=25$.

(2) Dynamic eddy-viscosity model (DEV): planeaveraged formulation of the dynamic model, similar to the implementation by Piomelli: ${ }^{35}$

$\tau_{i j}-\frac{\delta_{i j}}{3} \tau_{k k}=-2 C_{e v} \Delta^{2}|\bar{S}| \bar{S}_{i j}, \quad C_{e v}(y, t)=-\frac{1}{2} \frac{\left\langle\mathcal{L}_{i j} M_{i j}\right\rangle}{\left\langle M_{i j} M_{i j}\right\rangle}$,

where the brackets denote plane-averaging.

(3) Dynamic mixed model (DMM): Mixed, onecoefficient model similar to that used by Zang, Street, and Koseff. ${ }^{18}$ The model is given by Eq. (3.6) (the entire SGS stress tensor is modeled, rather than the anisotropic part only); the coefficient $C_{e v}$ is obtained from Eq. (3.14), the brackets denoting local averaging over the test-filter cell.

(4) Lagrangian eddy-viscosity model (LEV): Localized, Lagrangian-averaged dynamic model, similar to the implementation by Meneveau et al.; ${ }^{21}$ the model is given by Eq. (4.2), with $C_{e v}=C_{e v}(\mathbf{x}, t)$ and the brackets representing Lagrangian averaging performed according to Eqs. (3.23) and (3.24).

(5) Lagrangian one-coefficient mixed model (LMI): The model is again given by Eq. (3.6), and it is similar to the one used by $\mathrm{Wu}$ and Squires, ${ }^{20}$ apart from a difference in the Lagrangian time-scale and in the filtering length; the coeffcient $C_{e v}$ is obtained from Eq. (3.14) using the Lagrangian averaging procedure. In order to avoid complex values for $T$, if $C_{e v}(\mathbf{x}, t)=0$ is reached, $I_{L M}^{n}$ is set equal to $I_{L N}^{n}$. The scale similar part is defined according to the consistent formulation proposed by Vreman et al. ${ }^{19}$

(6) Two-coefficient Lagrangian mixed model (LM2): Following Anderson and Meneveau ${ }^{22}$ the scale-similar part is proportional to the resolved turbulent stresses $\mathcal{L}_{i j}$. The two model coefficients are obtained from Eqs. (3.17) and (3.18) using the Lagrangian averaging procedure. In this case, the clipping required to avoid complex values of $T$ is performed by setting

$$
\mathcal{I}_{L M}^{n}=\frac{\mathcal{I}_{L N}^{n} \mathcal{I}_{M N}^{n}}{\mathcal{I}_{N N}^{n}} \text {. }
$$

Coarse DNS calculations that used no model were also carried out to evaluate whether the SGS model had a significant contribution. They will be denoted by CDS.

The top-hat filter was used for all explicit filtering operations required for the evaluation of the scale-similar model, and as test filter. The expressions employed by Zang et al. ${ }^{18}$ were used here. Filtering was performed in planes of homogeneity only, to avoid commutation errors. The test- 


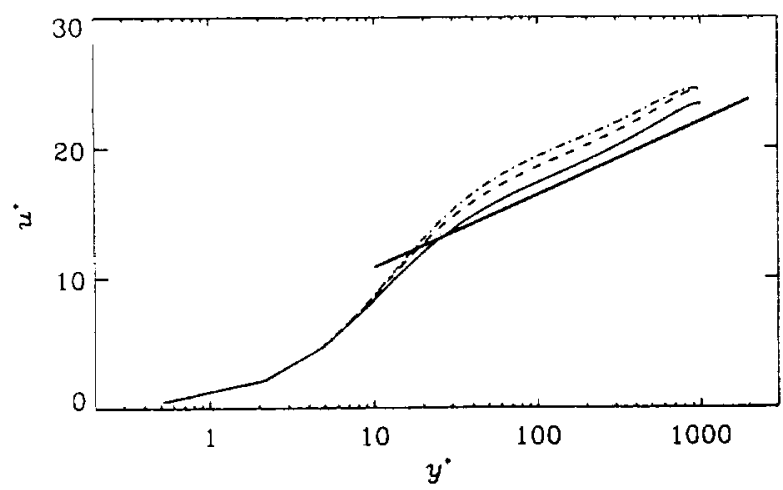

FIG. 2. Mean velocity profiles in wall coordinates in the two-dimensional plane channel, $\mathrm{Re}_{\tau}=1050$. Dynamic eddy-viscosity model (planeaveraged). - Fourier cutoff filter; $\cdots$ top-hat filter, $S_{i} / \bar{\Delta}_{i}=\sqrt{6}$; - - top-hat filter, $\Delta_{i} / \bar{\Delta}_{i}=2 ;-$ logarithmic law.

filter width in the $x$ and $z$ directions was chosen to be $\delta_{i}$ $=\sqrt{6 \Delta_{i}}$, following the work of Lund, ${ }^{34}$ who demonstrated that the use of a consistent filter width (determined based on the standard deviation of the discrete filter function) gives more accurate prediction of the turbulent statistics. This result was confirmed here: The mean velocity profile obtained with the inconsistent filter width $\Delta_{i}=2 \bar{\Delta}_{i}$, shown in Fig. 2 , are characterized by an excessively high wall stress, which results in a higher intercept of the logarithmic layer. It should be noted that the use of the top-hat filter rather than the Fourier cutoff, the natural choice when a spectral code is used, leads to a general decrease in the accuracy of the results, as also shown in Fig. 2. The choice of the top-hat filter in the present calculations was dictated by the desire to examine mixed models (with the Fourier cutoff filter $\overline{\bar{u}}_{i}=\bar{u}_{i}$ ); furthermore, present applications of LES to complex flows are bound to use finite-difference and finite-volume techniques, for which discrete filters similar to the top-hat filter are the natural choice.

\section{B. Two-dimensional channel flow}

The parameters of the 2D channel flow calculations were summarized in Table I. The wall stress obtained with the various models, at both Reynolds numbers examined, is reported in Table II. The most accurate prediction of the wall stress is obtained with the one-coefficient Lagrangian mixed model (LM1). The prediction of the wall stress is extremely sensitive to the SGS model used and, especially, to the spanwise grid resolution. In the present calculations the grid size

TABLE II. Wall shear stress.

\begin{tabular}{llc}
\hline & \multicolumn{2}{c}{$\tau_{w} \times 10^{3}$ (Error) } \\
& $\mathrm{Re}_{\tau}=180$ & 1.13 \\
\hline Experiment (Ref. 29) & 1.86 & $\ldots$ \\
SMG & $1.47(-20 \%)$ & $0.90(-20 \%)$ \\
DEV & $1.58(-15 \%)$ & $0.94(-16 \%)$ \\
DMM & $1.65(-12 \%)$ & $1.00(-11 \%)$ \\
LM1 & $1.66(-11 \%)$ & $1.32(+19 \%)$ \\
LM2 & $1.68(-10 \%)$ & $1.190)$ \\
\hline
\end{tabular}
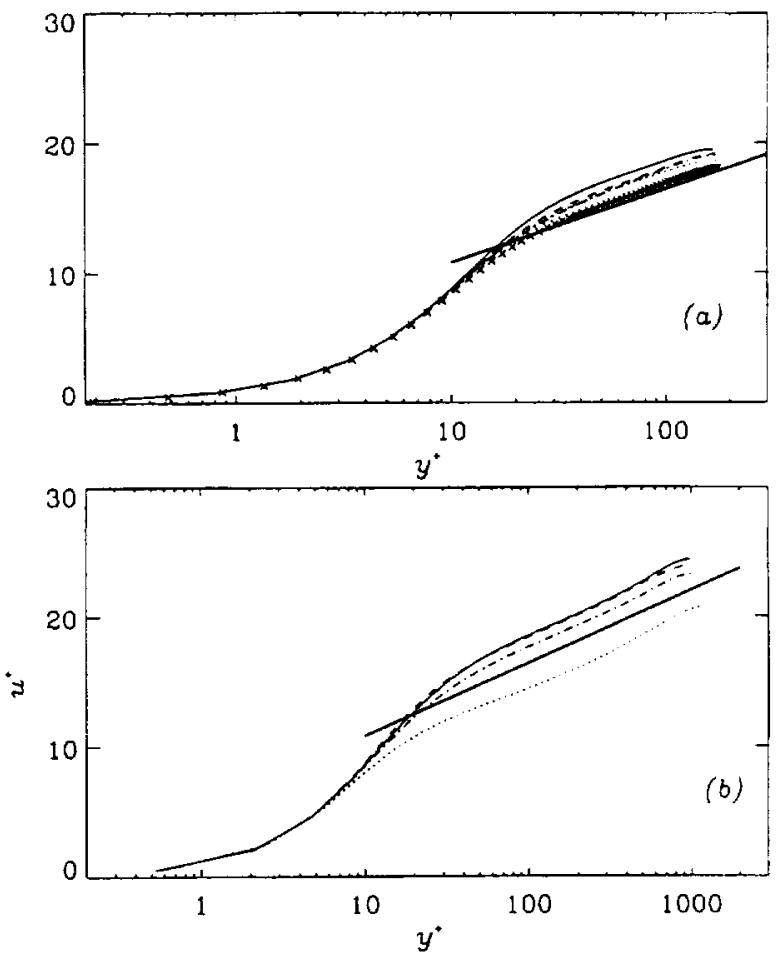

FIG. 3. Mean velocity profiles in wall coordinates in the two-dimensional plane channel. (a) $\operatorname{Re}_{\tau}=180$, (b) $\operatorname{Re}_{\tau}=1050 \times$ DNS data; - dynamic eddy-viscosity model; ... dynamic mixed model; - . - onecoefficient Lagrangian mixed model; .... two-coefficient Lagrangian mixed model; _ logarithmic law.

in the spanwise direction was $\Delta z^{+}=12$ and 26 , respectively, for the low and high Reynolds number calculations. The latter is barely sufficient to resolve the streaks, and some error in the prediction of the wall stress should be expected. Even when the Fourier cutoff filter is used, in fact, the wall stress is underpredicted by 5\%; use of the tophat filter, as shown in Fig. 2, amplifies this problem by increasing the model dissipation.

The mean velocity profiles, shown in Fig. 3, give the same indications: All models give a somewhat high intercept of the logarithmic layer, consistent with the low $\tau_{w}$, with the exception of the two-coefficient Lagrangian mixed model (LM2), which, at high Reynolds number, was found to be insufficiently dissipative, resulting in too high a value for $\tau_{w}$ and too low an intercept of the logarithmic layer.

All models predict turbulence intensities in good agreement with the DNS ${ }^{36}$ and experimental ${ }^{37}$ data. The streamwise intensities are shown in Fig. 4; similar results are obtained for the other components.

The effect of the SGS model is significant, especially at the high Reynolds number. The contribution of the eddy viscosity term remains fairly small (less than $10 \%$ of the wall stress, Fig. 5) especially with the two-coefficient mixed model LM2. The contribution of the scale-similar part of the model is larger, often significantly, than the eddy-viscosity one. The two mixed models yield comparable values of the total SGS shear; the dynamic mixed model (DMM) has a significantly larger eddy-viscosity contribution, caused by spurious high values due to the lack of averaging, as will be 

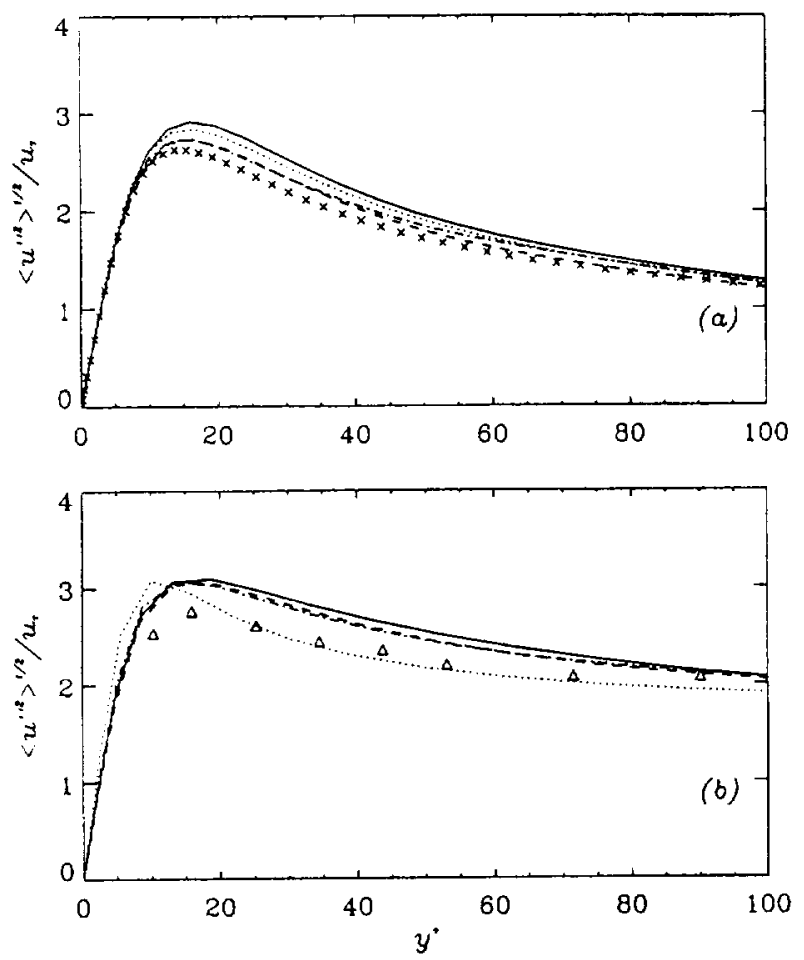

FIG. 4. Turbulence intensity profiles in wall coordinates in the twodimensional plane channel. (a) $\operatorname{Re}_{\tau}=180$, (b) $\mathrm{Re}_{\tau}=1050$. D Dynamic eddy-viscosity model; . ... dynamic mixed model; - . - onecoefficient Lagrangian mixed model; .... two-coefficient Lagrangian mixed model; $\times$ DNS data (Ref. 36); $\triangle$ experimental data (Ref. 37).

shown later; the fact that the total SGS stress is essentially the same as the Lagrangian mixed model (LM1), however, indicates that the scale-similar stresses adjust accordingly. In both models the scale-similar stresses have the same coeffcient $\left(C_{s s}=1\right)$; this indicates that the velocity spectra are being modified in a subtle manner as to maintain the total shear stress unchanged. In plane channel flow the plane- and time-averaged streamwise momentum equation

$$
\frac{\Delta P}{L}=\frac{d}{d y}\left(-\left\langle\bar{u}^{\prime} \bar{v}^{\prime}\right\rangle-\left\langle\tau_{12}\right\rangle+\nu \frac{d U}{d y}\right),
$$

(where $\langle\cdot\rangle$ denotes plane- and time-averaging, and a prime indicates a fluctuating quantity, $f^{\prime}=f-\langle f\rangle$ ) forces a balance between the SGS and resolved stresses (above $y^{+} \simeq 20$ the viscous contribution is negligible), that cannot change independently from each other.

The different behavior of the mixed models is also reflected in different energy interchange mechanisms. In Figs. 6 and 7 the contributions of the eddy-viscosity and scalesimilar parts of the SGS model to the SGS dissipation are shown. The SGS dissipation, defined here as $\epsilon_{\mathrm{sgs}}=-\tau_{i j} \bar{S}_{i j}$, represents the net large-scale energy drained by the subgrid scales; forward scatter (energy transfer to the small scales) corresponds to $\epsilon_{\mathrm{sgg}}>0$, backscatter (energy feedback from the small to the large scales) to $\epsilon_{\text {sgs }}<0$. In mixed models, two contributions to the SGS dissipation can be identified, one due to the eddy-viscosity part of the model, $\epsilon_{\mathrm{sgs}}^{e v}$ $=-2 C_{e v} \alpha_{i j} \bar{S}_{i j}$, the other to the scale-similar part, $\epsilon_{\mathrm{sgs}}^{s s}$
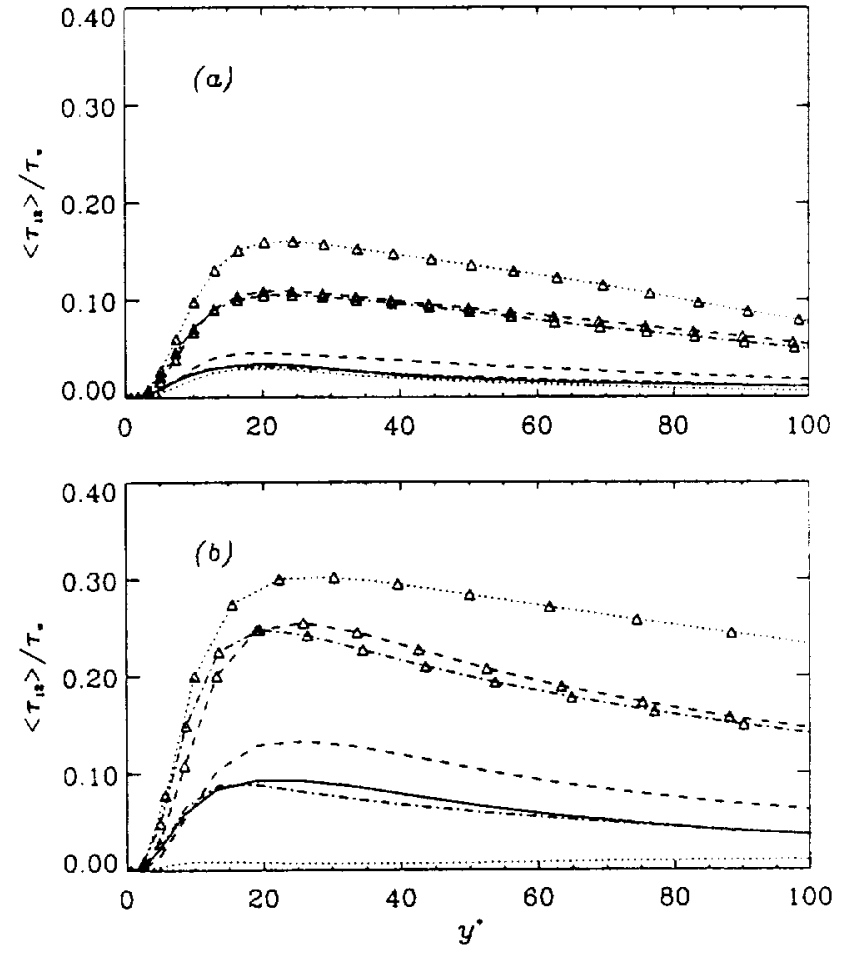

FIG. 5. Subgrid-scale shear stress in the two-dimensional plane channel. (a) $\operatorname{Re}_{T}=180$, (b) $\operatorname{Re}_{\mathrm{T}}=1050$. Dynamic eddy-viscosity model; - - dynamic mixed model; - - - one-coefficient Lagrangian mixed model; . . . Lagrangian two-coefficients mixed model. Lines: eddy viscosity component; lines and symbols: eddy viscosity and scale-similar components.

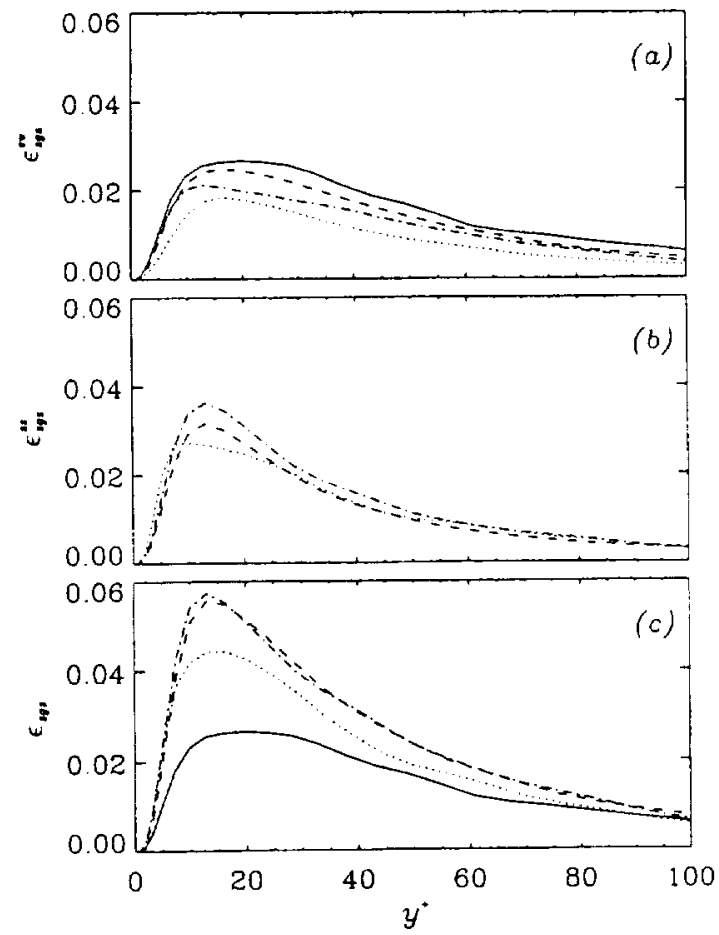

FIG. 6. Subgrid-scale dissipation in the two-dimensional plane channel, $\operatorname{Re}_{\tau}=180$. (a) Dissipation by the eddy-viscosity part of the SGS stresses, $\epsilon_{\mathrm{sgs}}^{e v} ;$ (b) dissipation by the scale-similar part of the SGS stresses, $\epsilon_{\mathrm{sgs}}^{s s} ;$ (c) total SGS dissipation, $\epsilon_{\mathrm{sgs}}$. - Dynamic eddy-viscosity model; - .. dynamic mixed model; - - - one-coefficient Lagrangian mixed model: .... two-coefficient Lagrangian mixed model. 


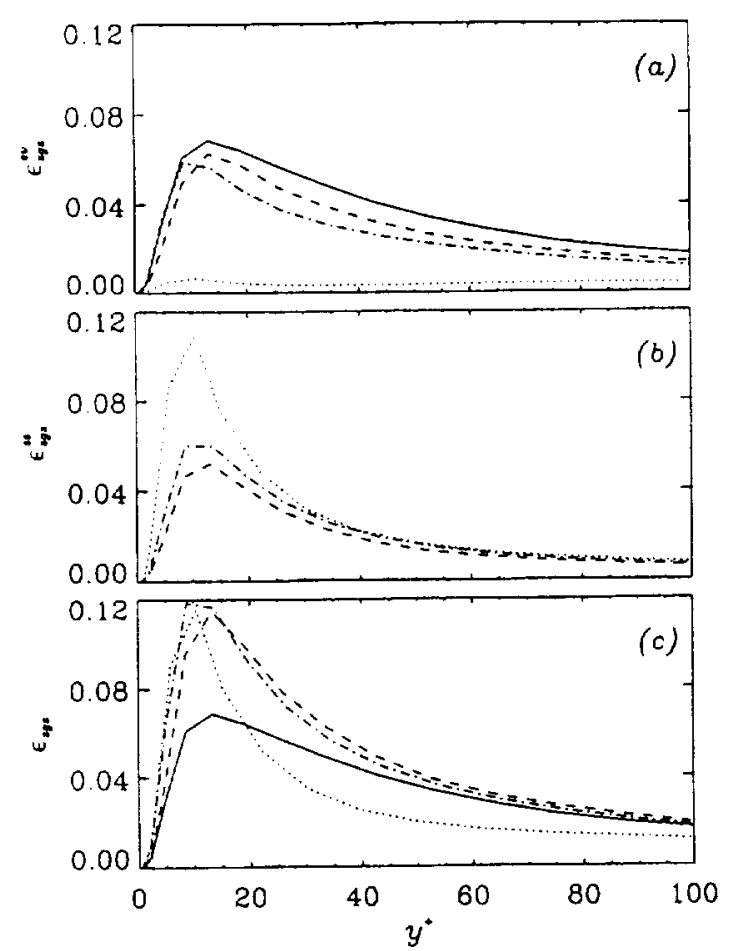

FIG. 7. Subgrid-scale dissipation in the two-dimensional plane channel, $\mathrm{Re}_{\tau}=1050$. (a) Dissipation by the eddy-viscosity part of the SGS stresses, $\epsilon_{\mathrm{sgs}}^{e v} ;$ (b) dissipation by the scale-similar part of the SGS stresses, $\epsilon_{\mathrm{sgs}}^{s s} ;$ (c) total SGS dissipation, $\epsilon_{\mathrm{sgs}}$. - Dynamic eddy-viscosity model; -.. dynamic mixed model; - . - one-coefficient Lagrangian mixed model; two-coefficient Lagrangian mixed model.

$=C_{s s} A_{i j} \bar{S}_{i j}$. At the low Reynolds number, Fig. 6, the two mixed models behave in a very similar manner; reflecting the differences in $\tau_{12}$, the eddy-viscosity component is larger for the dynamic mixed model, but the scale-similar parts adjust in such a way that the total SGS dissipation is nearly identical [Fig. 6(c)]. The dynamic eddy-viscosity model has the largest eddy-viscosity contribution, but the total $\epsilon_{\text {sgs }}$ is the smallest. The two-coefficient Lagrangian mixed model, LM2, is the one with the smallest eddy-viscosity dissipation.

At the higher Reynolds number, Fig. 7, the same behavior is observed for all models, with the exception of the two-coefficient Lagrangian mixed model, LM2, which exhibits a significant increase in the scale-similar contribution, partially balanced by a large decrease in the eddy-viscosity component. The total SGS dissipation is the lowest among the models examined, for $y^{+}>20$. The significant difference in the scale-similar and eddy-viscosity components of the SGS stresses and dissipation at the two Reynolds numbers is due to very different values of the model coefficients. At the high Reynolds number, $C_{s s}$ is three to four times higher than in the low Reynolds number case in the near-wall and buffer layers (Fig. 8). A decrease of $C_{e v}$ corresponds to this increase. It is difficult to determine whether the eddy-viscosity component of the decreases to adjust for the increase in the scale-similar part, or vice versa. The fact that the high contribution of the scale-similar part is due to an increased coefficient, rather than to an increase of the resolved turbulent stresses $\mathcal{L}_{i j}$, indicates that the dynamic procedure might be giving suspect results in this case. This may be due to the

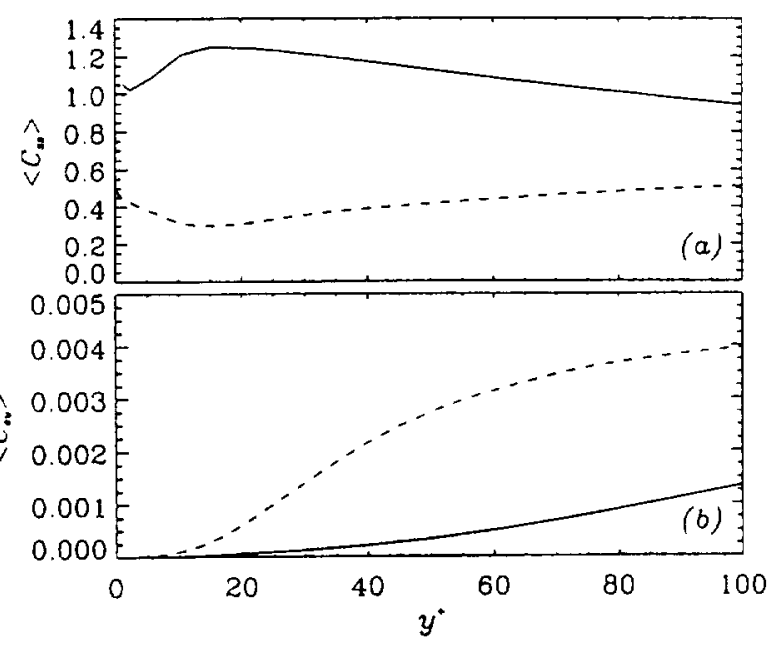

FIG. 8. Eddy-viscosity and scale-similar model coefficients in the twodimensional plane channel. Two-coefficient Lagrangian mixed model. $\operatorname{Re}_{\tau}=1050 ; \cdots \operatorname{Re}_{\tau}=180$. (a) $C_{\text {is }}$; (b) $C_{e v}$

fact that the two-coefficients Lagrangian mixed model LM2 requires three levels of filtering, the principal (grid) filter, the test filter, and a coarser one necessary to evaluate $B_{i j}$. With the resolution of the high Reynolds number calculation, the last one corresponds to a filter width equal to 620 wall units in the streamwise direction, 156 in the spanwise direction. Such a filter width is significantly larger than most of the eddies that govern the momentum and energy transfer in the buffer layer. At the low Reynolds number, on the other hand, the coarsest filter has a more reasonable width of 282 wall units in $x$ and 72 in $z$.

The Lagrangian ensemble-averaging procedure is very effective in removing spurious oscillations of the eddyviscosity coefficient, as observed by Meneveau et al., ${ }^{21}$ even in mixed models. Figure 9 compares the average, root-meansquare (rms) and maximum values of the eddy-viscosity coefficient, $C_{e u}$, for the dynamic eddy-viscosity model, DEV, the dynamic mixed model, DMM, and the two Lagrangian mixed models, LM1 and LM2. The values obtained using the plane-averaged DEV model are consistent with previous results: $C_{e v}$ approaches the Smagorinsky constant value, $C_{s}^{2}$ $=0.01$ towards the center of the channel, but decays rapidly (like $y^{+3}$ ) near the wall. With the Lagrangian averaging, the values of the rms and maximum $C_{e u}$ are of the same order as the average. As mentioned previously, the dynamic mixed model gives a higher value of the coefficient; this is due to the fact that, following Zang et al., ${ }^{18}$ the only averaging performed here was over the test-filter size; this results in spuriously high values of the coefficient, as confirmed by the rms and maximum values of the coefficient which are, respectively, twenty and fifty times higher than the mean value. These oscillations, however, do not cause numerical instabilities (partly because the most severe ones occur near the center of the channel, where the shear is small). It is conceivable that in finite-volume codes (especially those that use upwind schemes such as the one used by Zang et al. ${ }^{18}$ ) the velocity spectra might decay more rapidly than in the present case, leading to smoother fields of $C_{e v}$. 


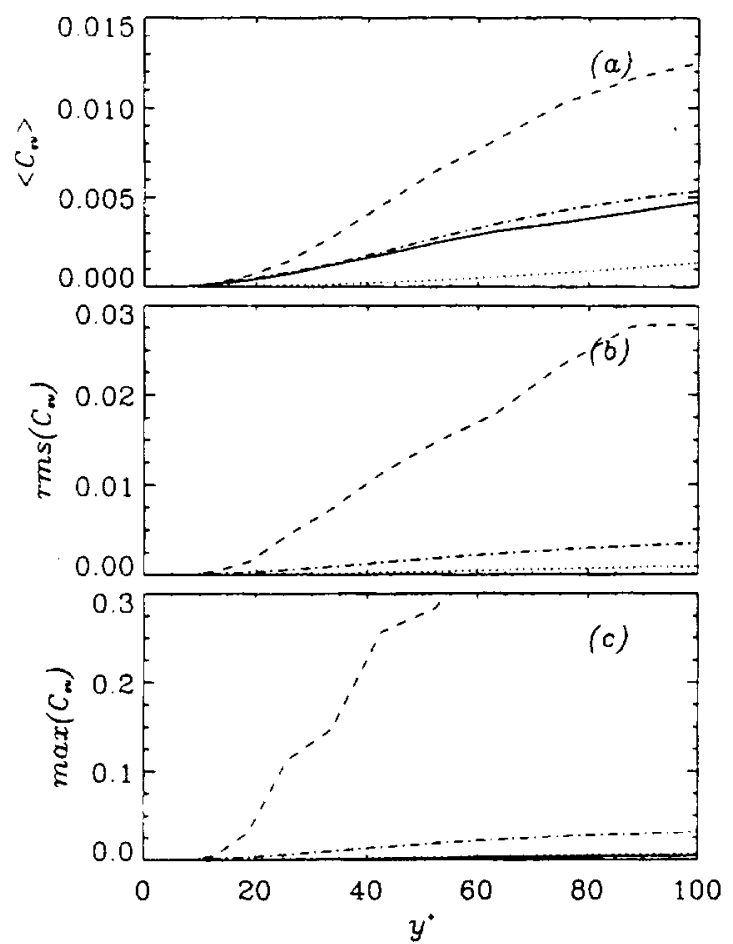

FIG. 9. Eddy-viscosity model coefficient in the two-dimensional plane channel, $\mathrm{Re}_{\mathrm{\gamma}}=1050$. - Dynamic eddy-viscosity model; - - dynamic mixed model; - . - one-coefficient Lagrangian mixed model; . . . two-coefficient Lagrangian mixed model. (a) average; (b) root-mean-square; (c) maximum.

\section{Three-dimensional boundary layer}

A completely different physical scenario occurs in the three-dimensional boundary layer, in which local nonequilibrium effects are significant. In Fig. 10 the evolution of the wall-shear stress, $\tau_{w}$, and the turbulent kinetic energy aver-

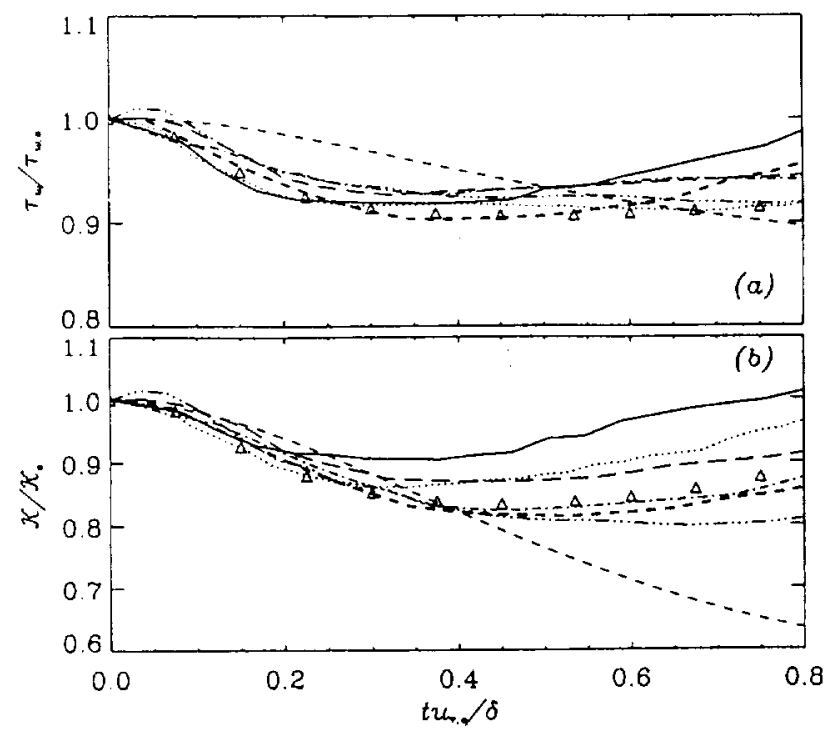

FIG. 10. Time history of the wall shear stress and average turbulent kinetic energy in the three-dimensional boundary layer flow. All quantities are initialized by their initial values. (a) $\tau_{w} / \tau_{w . u}$; (b) $\mathcal{K} / K_{0}$. - Coarse DNS; -. Smagorinsky model; - . - dynamic eddy-viscosity model; ... dynamic mixed model; - . . - one-coefficient Lagrangian mixed model; ... (wo-coefficient Lagrangian mixed model; $\triangle$ DNS (Ref. 27).

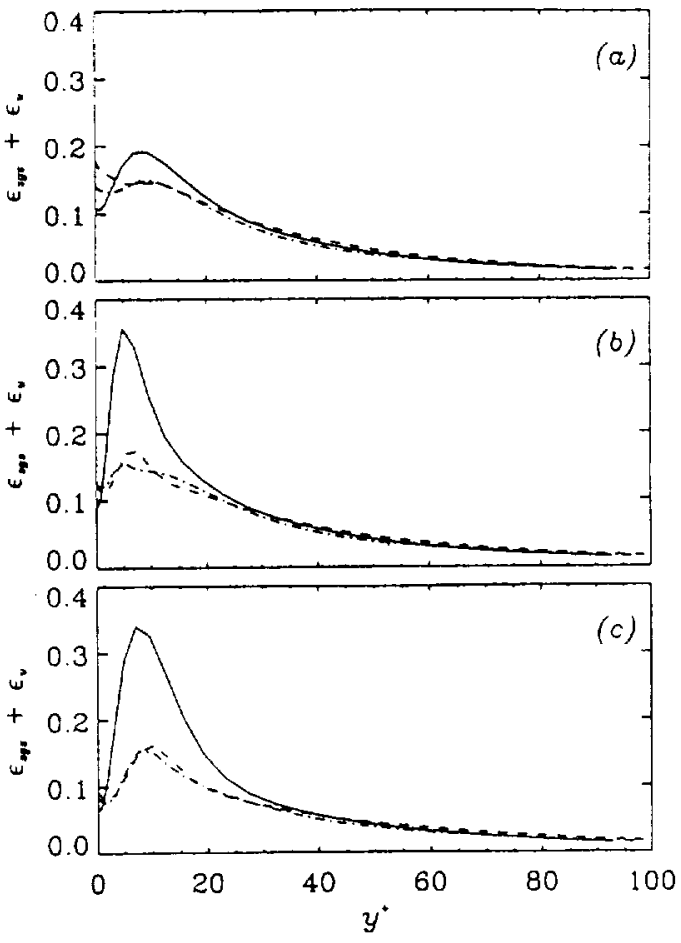

FIG. 11. Total (viscous +SGS) dissipation profiles. - Smagorinsky model; .. - dynamic eddy-viscosity model; - . - one-coefficient Lagrangian model. (a) $t / u_{T, o} \leqslant 0 ;$ (b) $t / u_{T, o}=0.08$; (c) $t / u_{\tau, o}=0.16$.

aged over the entire channel height, $\mathcal{K}$, are shown as a function of dimensionless time $t u_{\tau, o} / \delta$. Both quantities are normalized by their initial values, $\tau_{w, o}$ and $\mathcal{K}_{0}$.

Although at this Reynolds number the SGS stresses are smaller than at $\operatorname{Re}=1050$, the effect of the model is significant. Computations performed without any model (CDS), in fact, are unable to predict the nonequilibrium effects, and give the incorrect time development of all the quantities examined. The Smagorinsky model also gives significant errors: Before the shear is applied, the model over-predicts the dissipation slightly, especially in the buffer region. The imposition of the strain leads to a doubling of the SGS dissipation (Fig. 11), that results in excessive damping of the turbulent fluctuations .

Other models (DEV and LM1, for instance) have a less abrupt change in the dissipation, due to the fact that the model itself adjusts to the imposition of the shear: In Fig. 12 the time development of the plane-averaged coefficient is shown at three locations in the layer. Near the wall, the sudden appearance of $d W / d y$ results in an increased value of the strain-rate magnitude $|\bar{S}|$, but also in an increase of the denominator of Eq. (3.14), and, hence, in a decrease of the magnitude of the coefficient that counteracts the increase of $|\bar{S}|$. Notice how the decrease of $C_{e v}$ occurs later at $y_{o}^{+} \simeq 13$ than at $y_{0}^{+}=6$, and not at all at $y_{0}^{+}+\approx 57$, reflecting the time required by the disturbance to propagate away from the wall. The self-adjusting character of the dynamic models led to improved prediction of the response of the flow to the perturbation. The one-coefficient Lagrangian mixed model, LM1, predicts the turbulent kinetic energy development very accurately, the wall stress only slightly less so. The two- 


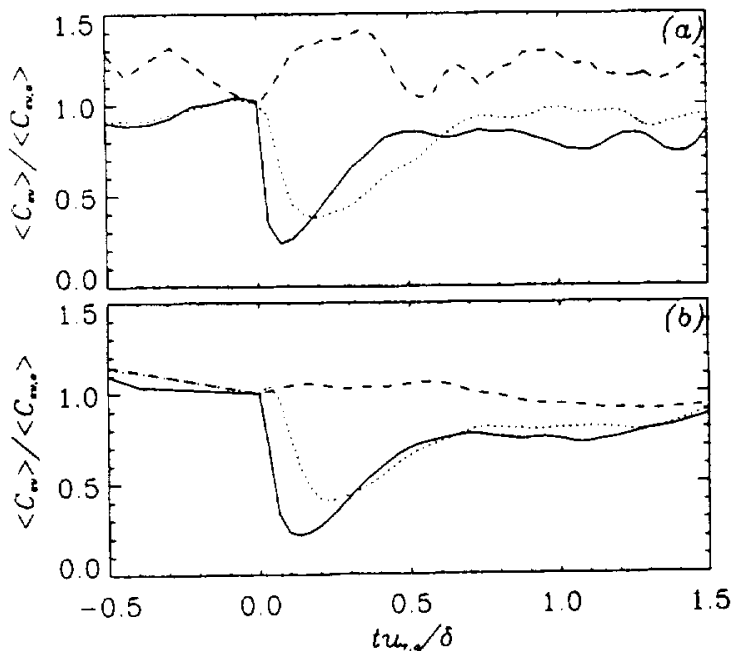

FIG. 12. Time-development of the coefficient $C_{e v}$ in the three-dimensional boundary layer flow. $-y_{o}^{+} \approx 6 ;-\cdots y_{o}^{+} \approx 13 ; \cdots y_{0}^{+}=57$. (a) Dynamic eddy-viscosity model; (b) one-coefficient Lagrangian mixed model.

coefficient Lagrangian mixed model, LM2, also gives accurate results.

Very little difference can be observed between the mean velocity profiles obtained with the various models (Fig. 13). A more significant difference is apparent in the prediction of the turbulent kinetic energy $\mathcal{K}$, shown in Fig. 14. The results obtained with the Lagrangian eddy-viscosity model, LEV, are generally more accurate than those yielded by the planeaveraged model DEV, confirming the positive features of this technique. The introduction of a scale-similar term, how-
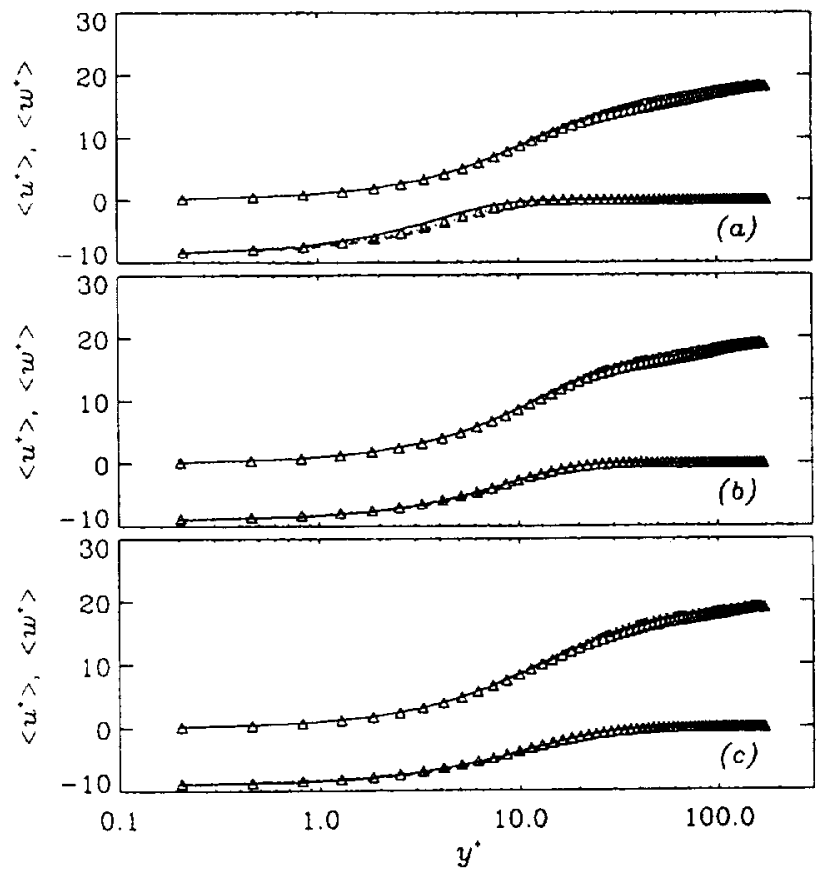

FIG. 13. Mean velocity profiles in the three-dimensional boundary layer flow. (a) $t u_{\tau, v} / \delta=0.075$; (b) $t u_{T, d} / \delta=0.3$; (c) $t u_{\tau, 0} / \delta=0.6$. D Dynamic eddy-viscosity model; -.. dynamic mixed model; - . - onecoefficient Lagrangian mixed model; .... two-coefficient Lagrangian mixed model; $\triangle$ DNS (Ref. 27).

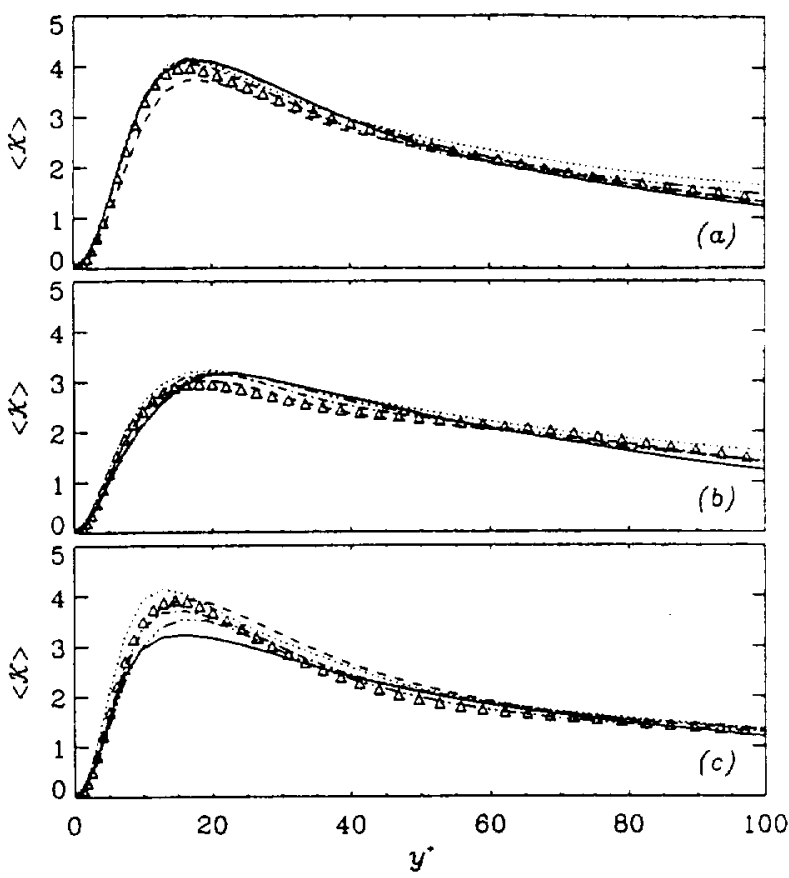

FIG. 14. Turbulent kinetic energy profiles in the three-dimensional boundary layer flow. (a) $t u_{\tau, o} / \delta=0.075$; (b) $t u_{\tau, o} / \delta=0.3$; (c) $t u_{\tau, o} / \delta=0.6$. Dynamic eddy-viscosity model; ... dynamic mixed model; - . one-coefficient Lagrangian mixed model; $\ldots$... two-coefficient Lagrangian mixed model; $\triangle$ DNS (Ref. 27).

ever, appears to be beneficial, since the three mixed models tested give consistently better results than either the purely dissipative ones.

Particularly important, in this flow, is an accurate prediction of the secondary stresses $\left\langle u^{\prime} w^{\prime}\right\rangle$ and $\left\langle v^{\prime} w^{\prime}\right\rangle$. Although both are significant, the former can become significantly larger than the primary stress, $\left\langle u^{\prime} v^{\prime}\right\rangle$. The mixed models, and in particular the one-coefficient Lagrangian mixed model LM1, predict the secondary stresses very accurately (Fig. 15).

\section{CONCLUSIONS}

The predictions of three mixed models have been compared with those of three eddy-viscosity models, and with DNS and experimental data, in two flows, a two-dimensional plane channel, both at low and moderately high Reynolds numbers, and a three-dimensional boundary layer obtained by moving the wall of a fully-developed plane channel in the spanwise direction.

In general, mixed models are found to give more accurate results than eddy-viscosity ones. For the dynamic and one-coefficient Lagrangian mixed models (DMM and LM1) this might be due to the fact that using these model is equivalent to filtering the Navier-Stokes equations explicitly. Lund $^{34}$ discussed the advantages of such procedure, which has the drawback that Galilean invariance is lost unless a scale-similar model of the form Eq. (3.5) with a coefficient equal to one is used. Lund ${ }^{34}$ expressed some concem that the use of a scale-similar model might introduce higher frequencies into the calculation and lead to less accurate results. This potential problem was not observed here, even when the dy- 


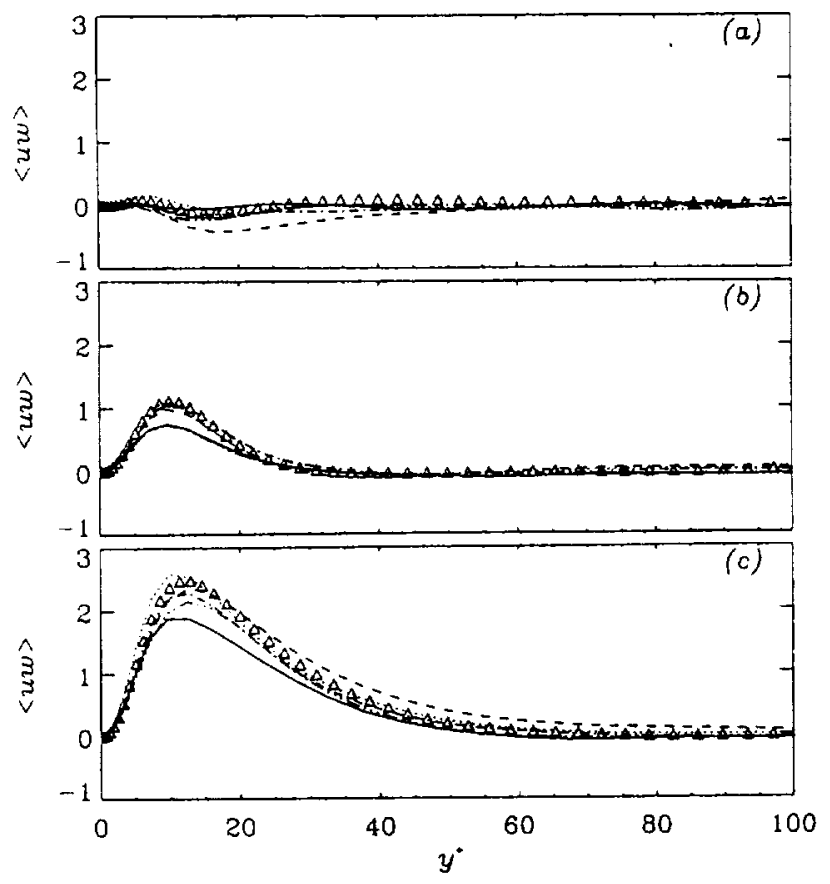

FIG. 15. $\left\langle u^{\prime} w^{\prime}\right\rangle$ shear stress profiles in the three-dimensional boundary layer flow. (a) $t u_{r, o} / \delta=0.075$; (b) $t u_{r, o} / \delta=0.3$; (c) $t u_{\tau, o} / \delta=0.6$. D Dynamic eddy-viscosily model; - - L Lagrangian eddy-viscosity model; ... dynamic mixed model; - - - one-coefficient Lagrangian mixed model; . . . . two-coefficient Lagrangian mixed model; $\triangle$ DNS (Ref. 27).

namic mixed model, DMM, was used; this model gave improved results over the plane-averaged eddy-viscosity model in all the cases examined, although only very significant fluctuations of the model coefficient were present (which, however, did not lead to numerical instabilities).

The Lagrangian averaging procedure was found to be extremely beneficial, giving in general better results than either plane or local averaging. The value of the time constant $T$ recommended by Meneveau et al. ${ }^{21}$ was used here for the eddy-viscosity term and (in the case of the two-coefficient mixed model, LM2) for the scale-similar part as well. The results obtained with the LM2 model were not, altogether, satisfactory: While at low Reynolds number the model performed well, both for the two-dimensional and threedimensional calculations, the results obtained at the higher Reynolds number were not particularly accurate. Two possible reasons for this decrease in accuracy are the fact that the LM2 model requires three levels of filtering, $\bar{\Delta}, \Delta$ $=\sqrt{6 \Delta}$, and $\bar{\Delta}=6 \bar{\Delta}$. At high Reynolds numbers the coarsest filter is clearly in the energy-containing range, and the assumptions on which the dynamic procedure is based (primarily, that of similarity of the stresses) are expected to fail. It is also possible that the use of a different time constant $T$ in the Lagrangian averaging for the terms that involve the scale-similar terms might be required (since those represent interaction between scales of similar size, while the eddyviscosity term models the distant interactions). It is unlikely that a Reynolds-number dependence of $T$ might be the principal cause of error, since the higher Reynolds numbers calculation was performed at a value of $\mathrm{Re}_{\tau}$ only $50 \%$ higher than the value of 640 computed by Meneveau et al. ${ }^{21}$
TABLE III. CPU time required by various models, normalized by that used by the Smagorinsky model.

\begin{tabular}{ll}
\hline \hline \multicolumn{1}{c}{ Model } & CPU \\
\hline No model (CDS) & 0.93 \\
Smagorinsky (SMG) & 1.00 \\
Dynamic eddy viscosity (DEV) & 1.07 \\
Dynamic mixed (DMM) & 1.11 \\
Lagrangian eddy viscosity (LEV) & 1.20 \\
Lagrangian mixed one-coefficient (LM1) & 1.25 \\
Lagrangian mixed two-coefficient (LM2) & 1.31 \\
\hline \hline
\end{tabular}

One important consideration regards the increased resources required by more complex LES models. The dynamic model requires more operations and memory than the Smagorinsky one, since the various terms used in the contractions must be computed and stored. Mixed models and the Lagrangian averaging procedure require additional effort. Table III reports the CPU time (normalized by the Smagorinsky model) of each model. The numbers reported show, of course, only qualitative trends, since they are strongly implementation- and machine-dependent. In this particular case, all the computations were run on a Cray C90, and the model coefficient (or coefficients) were evaluated once per step, while both the eddy-viscosity and the scale-similar models were computed at each Runge-Kutta stage. It is quite readily seen that the additional cost of the Lagrangian models is significant.

The increased CPU time may be partly due to the difficulty in vectorizing the Lagrangian interpolation routines. The CPU time used by the dynamic mixed model, DMM, is actually substantially higher than apparent in Table III, since, due to the fluctuations of the coefficient $C_{e v}$, the time step had to be decreased by $25 \%$ to avoid numerical instabilities. Altogether, the additional effort required by the onecoefficient mixed model, LM1, may be justified by its higher accuracy. The use of additional filters, or the evaluation of additional model coefficients might make the cost of the model excessive, and might not be worth the computational effort.

The one-coefficient Lagrangian mixed model (LM1) was the one that gave, in our opinion, the best results, on the average, among those tested. Compared with the dynamic mixed model (DMM) it was more stable and cost effective, and more likely to be accurate when extended to more complex configurations.

\section{ACKNOWLEDGMENTS}

The support by the Office of Naval Research under Grant No. N-00014-91-J-1638 monitored by Dr. L. Patrick Purtell, and by the NASA under Grant Nos. NAG 1-1828 monitored by Dr. Craig L. Streett and NAG 1-1880, monitored by Dr. Michele G. Macaraeg is gratefully acknowledged. The CPU time was provided by the High Performance Computing program.

'J. Smagorinsky, "General circulation experiments with the primitive equations. I. The basic experiment," Mon. Weather Rev. 91, 99 (1963).

${ }^{2}$ R. A. Clark, J. H. Ferziger, and W. C. Reynolds, "Evaluation of subgrid 
scale modets using an accurately simulated turbulent flow," J. Fluid Mech. 91, 1 (1979).

${ }^{3}$ U. Piomelli, P. Moin, and J. H. Ferziger, "Model consistency in large eddy simulation of turbulent channel flows," Phys. Fluids 31, 1884 (1988).

${ }^{4}$ E. R. Van Driest, "On the turbulent flow near a wall," J. Aeronaut. Sci. 23. 1007 (1956).

${ }^{5}$ U. Piomelli, T. A. Zang, C. G. Speziale, and M. Y. Hussaini, "On the large-eddy simulation of transitional wall-bounded flows," Phys. Fluids A 2, 257 (1990).

${ }^{6} \mathrm{P}$. Voke and Z. Yang, "Numerical study of bypass transition," Phys. Fluids 7. 2256 (1995).

${ }^{7}$ M. Germano, U. Piomelli, P. Moin, and W. H. Cabot, "A dynamic subgrid-scale eddy viscosity model," Phys. Fluids A 3, 1760 (1991).

${ }^{8} \mathrm{D}$. K. Lilly, "A proposed modification of the Germano subgrid-scale closure method," Phys. Fluids A 4, 633 (1992).

${ }^{9}$ R. H. Kraichnan, "Eddy viscosity in two and three dimensions," J. Atmo. Sciences 33, 1521 (1976).

${ }^{10}$ U. Piomelli, W. H. Cabot, P. Moin, and S. Lee, "'Subgrid-scale backscatter in turbulent and transitional flows," Phys. Fluids A 3, 1766 (1991).

"S. Liu, C. Meneveau, and J. Katz, "On the properties of similarity subgrid-scale models as deduced from measurements in a turbulent jet," J. Fluid Mech. 275, 83 (1994).

${ }^{12}$ S. Ghosal, T. S. Lund, P. Moin, and K. Akselvoll, "A dynamic localization model for large-eddy simulation of turbulent flow," J. Fluid Mech. 286, 229 (1995)

${ }^{13}$ J. A. Domaradzki, W. Liu, and M. E. Brachet, "An analysis of subgridscale interactions in numerically simulated isotropic turbulence," Phys. Fluids A 5, 1747 (1993).

${ }^{14} \mathrm{~J}$. A. Domaradzki, W. Liu, C. Härtel, and L. Kleiser, "Energy transfer in numerically simulated wall-bounded turbulent flows," Phys. Fluids 6 , 1583 (1994)

${ }^{15}$ U. Piomelli, Y. Yu, and R. J. Adrian, "Subgrid-scale energy transfer and near-wall turbulence structure," Phys. Fluids 8, 215 (1996).

${ }^{16} \mathrm{~J}$. Bardina, J. H. Ferziger, and W. C. Reynolds, "Improved subgrid scale models for large eddy simulation," AIAA Pap. 80-1357 (1980).

${ }^{17} \mathrm{~K}$. Horiuti, "The role of the Bardina model in large eddy simulation of turbulent channel flow," Phys. Fluids A 1, 426 (1989).

${ }^{18}$ Y. Zang, R. L. Street, and J. Koseff, "A dynamic mixed subgrid-scale model and its application to turbulent recirculating flows," Phys. Fluids A 5, 3186 (1993).

${ }^{19}$ B. Vreman, B. Geurts, and H. Kuerten, "On the formulation of the dynamic mixed subgrid-scale model," Phys. Fluids 6, 4057 (1994).

${ }^{20} \mathrm{X}$. Wu and K. D. Squires, "Large eddy simulation of an equilibrium three dimensional turbulent boundary layer," AIAA J. 1, 67 (1997).

${ }^{21} \mathrm{C}$. Meneveau, T. S. Lund, and W. H. Cabot, "A Lagrangian dynamic subgrid-scale model of turbulence," J. Fluid Mech. 319, 353 (1996).

${ }^{22} \mathrm{R}$. Anderson and C. Meneveau, " Effects of the similarity model in finitedifference LES of decaying isotropic turbulence using a Lagrangian dynamic mixed model," 'Flow, Turbulence and Combustion (submitted).

${ }^{23} \mathrm{~V}$. M. Salvetti and S. Banerjee, "A prion tests of a new dynamic subgridscale model for finite-difference large-eddy simulations," Phys. Fluids 7 , 2831 (1995).

${ }^{24} \mathrm{~K}$. Horiuti, "A new dynamic two-parameter mixed model for large eddy simulation," Phys. Fluids 9, 3443 (1997).

${ }^{25}$ M. V. Salvetti, Y. Zang, R. L. Street, and S. Banerjee, "Large-eddy simulation of free-surface decaying turbulence with dynamic subgrid-scale models," Phys. Fluids 9, 2405 (1997).

${ }^{26} \mathrm{U}$. Piomelli, G. Coleman, and J. Kim, "On the effects of non-equilibrium on the subgrid-scale stresses," Phys. Fluids 9, 2740 (1997).

${ }^{27} \mathrm{G}$. K. Coleman, J. Kim, and A.-T. Le, "A numerical study of threedimensional boundary layers," Int. J. Heat Fluid Flow 17, 333 (1996).

${ }^{28}$ T. A. Zang and $M$. Y. Hussaini, "On spectral multigrid methods for the time-dependent Navier-Stokes equations," Appl. Math. Comput. 19. 359 (1986).

${ }^{29}$ R. B. Dean, "Reynolds Number Dependence of Skin Friction and Other Bulk Flow Variables in Two-Dimensional Rectangular Duct Flow," J. Fluids Eng. 100, 215 (1978).

${ }^{30} \mathrm{D}$. K. Lilly, "The representation of small-scale turbulence in numerical simulation experiments," in Proc. IBM Scientific Computing Symp. on Environmental Sciences (Yorktown Heights, N.Y., 195, 1967).

${ }^{31} \mathrm{C}$. G. Speziale, "Galilean invariance of subgrid scale stress models in the large eddy simulation of turbulence," J. Fluid Mech. 156, 55 (1985).

${ }^{32} \mathrm{M}$. Germano, "Turbulence: the filtering approach," J. Fluid Mech. 238, 325 (1992)

${ }^{33}$ It is assumed here that the coefficients, $C_{e v}$ and later $C_{s x}$ are smooth on the $\delta$ scale, and can, therefore, be extracted from the filtering operation.

${ }^{34} \mathrm{~T}$. S. Lund, "On the use of discrete filters for Large-eddy simulation," in Center for Turbulence Research Annual Briefs 1997 (Stanford University, Standford, 1998), p. 83.

${ }^{35} \mathrm{U}$. Piomelli, "High Reynolds number calculations using the dynamic subgrid-scale stress model," Phys. Fluids A 5, 1484 (1993).

${ }^{36} \mathrm{~J}$. Kim, P. Moin, and R. D. Moser, "Turbulence statistics in fully. developed channel flow at low Reynolds number," J. Fluid Mech. 177, 133 (1987).

${ }^{37} \mathrm{~T}$. Wei and W. W. Willmarth, "Reynolds number effects on the structure of a turbulent channel flow," J. Fluid Mech. 264, 57 (1989). 